\title{
Konzeptionsprobleme einer europäischen Geschichte des Mittelalters
}

\author{
(vorgetragen in der Plenarsitzung am 4. Juni 2010) \\ Rudolf SCHIEFFER
}

Nichts hat im 19. Jahrhundert die Erforschung und Darstellung der mittelalterlichen Geschichte so sehr beflügelt und zu gesellschaftlicher Anerkennung gebracht wie das nationale Bewußtsein. Die gemeinsame Vergangenheit der Deutschen so weit wie irgend möglich zurückzuverfolgen, die Eigenart ihres historischen Weges zu ergründen und dabei die Unterschiede zu anderen Nationen zu erkennen, war von höchstem allgemeinen Interesse. Führende Historiker damaliger Zeit begriffen sich als Wegbereiter und Lehrmeister der unter großen politischen Mühen zustande gebrachten deutschen Einheit und prägten ein suggestives Geschichtsbild, das alle Sympathie auf die monarchische Zentralgewalt der mittelalterlichen Kaiser und Könige lenkte und dementsprechend kritisch mit den Gegenkräften umging, also dem eigensüchtigen Partikularismus der Fürsten und erst recht den anmaßenden Eingriffen der Päpste. Das war nicht allein in Deutschland so, auch alle anderen europäischen Nationalbewegungen des 19. und des 20. Jahrhunderts, die griechische wie die belgische, die irische wie die serbische, fußten auf der von Historikern geschürten Wunschvorstellung von einem über weiteste Zeiträume mit sich identisch gebliebenen Staatsvolk, das sich auch nach jahrhundertelanger Fremdherrschaft den Weg zu politischer Eigenständigkeit zu bahnen bestimmt war. Das Mittelalter wurde zum Wurzelgrund der nationalen Selbstbehauptung.

Historische Umbrüche des 20. Jahrhunderts, die ich nicht näher zu schildern brauche, haben dazu geführt, daß zumal in Deutschland, aber keineswegs nur hierzulande, der politische Leitstern der Nation als einer zeitlosen, gewissermaßen apriorischen Größe erheblich an Strahlkraft eingebüßt hat und demgemäß auch die wissenschaftliche Kritik an der undifferenzierten Konstruktion einer deutschen Geschichte seit Arminius, einer französischen seit Vercingetorix, einer italienischen gar seit Romulus und Remus beherrschend geworden ist. An die Stelle der Fixierung auf die Nation, die meines Erachtens wegen ihrer neuzeitlichen Bedeutung stets ein legitimer Gegenstand historischer Reflexion bleiben wird, sind vielfältige andere Horizonte 
der Historiker getreten, unter denen Europa infolge der politischen Entwicklung nach 1945 und mehr noch seit 1989/90 besonders viele Blicke auf sich zieht.

Sich dem gesteigerten Bedürfnis nach Orientierung über das historisch bedingte Profil unseres Kontinents, über seinen unverwechselbaren Platz in der Welt von gestern und von morgen zu stellen, also europäische Geschichte zu schreiben, sollte nicht als plumpe Anbiederung an den Zeitgeist, als Vereinnahmung durch die jeweils aktuelle Politik beargwöhnt werden, sondern entspricht, wie ich finde, der genuinen Aufgabe des Historikers, seine wissenschaftlich gewonnene Expertise für den gesellschaftlichen Diskurs fruchtbar werden zu lassen. Welche Gefahren dabei tunlichst zu vermeiden sind, kann der Rückblick auf den Überschwang der nationalen Geschichtsbetrachtung früherer Generationen zeigen. So wird es nicht darum gehen dürfen, die politische Einigung der europäischen Völker oder auch bestimmte Konzepte ihrer Einheit vermeintlich zwingend aus den Tiefen der Vergangenheit abzuleiten; vielmehr muß, wie wir es bei der Geschichte der Nationen gelernt haben, auch in diesem Falle die Offenheit des historischen Prozesses betont werden, der in allen Stadien integrierende ebenso wie desintegrierende Faktoren aufweist und von den jeweils handelnden Zeitgenossen nicht in derselben Weise wahrgenommen wurde wie aus der generalisierenden, kategorisierenden Sicht des heutigen um Europa bemühten Historikers. Dieses Europa war so wenig wie seine einzelnen Völker eine historische Größe, für die eine zeitlose Identität beansprucht werden kann, sondern wie alles auf der Welt dem Gesetz des historischen Wandels unterworfen, der es nicht leicht macht, einen roten Faden für seine Darstellung herauszupräparieren.

Natürlich ist es nicht so, als ob der europäische Horizont erst neuerdings von der deutschen (wie auch der internationalen) Mediävistik in den Blick gefaßt worden wäre. Bei wesentlichen Themenfeldern wie der Geschichte des Papsttums und der religiösen Bewegungen, der Kreuzzüge und der Feudalgesellschaft, des Bildungswesens und der literarischen Kultur, des römischen und des kanonischen Rechts lag es seit jeher auf der Hand, daß sie sich ganz überwiegend einer bloß nationalen Betrachtungsweise entziehen. So sind in diesen Bereichen vor und nach 1945 bedeutende Forschungen und Synthesen vorgelegt worden, die sich zwar strenggenommen nicht auf den gesamten Kontinent im geographischen Sinne bezogen, aber doch vom Bewußtsein getragen waren, einen gemeinsamen Wurzelgrund der Völker des lateinisch-christlichen Europa im Mittelalter aufzudecken. Anders waren und sind schon von der Sache her die Bedingungen bei der Geschichte der Könige und ihrer Reiche, die seit dem Zerfall des regnum 
Francorum im 9./10. Jahrhundert wie schon zur Völkerwanderungszeit wieder in der Mehrzahl auftraten und bis ins Spätmittelalter ständig an Zahl zunahmen, aber sich erst mit Beginn der Neuzeit zu einem funktionellen „europäischen Staatensystem“ formierten. Als dessen spezifisch mittelalterliche Vorstufe hat man zumal von deutscher Seite gern die Vorrangstellung des römisch-fränkischen, später römisch-deutschen Kaisertums oder auch das Nebeneinander von lateinischem und griechischem Imperium aufgefaßt, doch handelt es sich dabei um Phänomene von bloß relativer und im Laufe der Jahrhunderte rapide abnehmender Tragweite, worauf sich schwerlich ein überzeugendes politikgeschichtliches Gesamtbild des europäischen Mittelalters gründen ließe. So kommt es, daß gerade in dieser zentralen Hinsicht bis heute eine individualisierend-nationalgeschichtliche Optik vorherrscht und eine gesonderte Behandlung nach Einzelreichen nahelegt, sobald es darum gehen soll, die politische Entwicklung Europas im Mittelalter nachzuzeichnen.

Ich lasse dahingestellt, inwieweit auch künftig Bedarf nach solchen additiven Entwürfen eines „Europa der Vaterländer“ besteht, und wende mich der Frage zu, ob sich im Lichte der jüngsten Fachdiskussion andere Ansatzpunkte denken lassen, um der Totalität des mittelalterlichen Europa darstellerisch besser gerecht zu werden.

Nach den Erfahrungen mit dem Bemühen, eine vertretbare Eingrenzung für die Geschichte der Deutschen zu finden, die erst vom 11. Jahrhundert an in nennenswerter Häufigkeit als Theutonici begrifflich in Erscheinung treten, zuvor also mangels spezifischer Fremd- und Selbstbezeichnung kaum als eine ihrer selbst bewußte historische Größe anzusehen sind, könnte es lohnen, von der Verbreitung und dem Sinngehalt des Terminus Europa auszugehen, der ebenso wie übrigens Germania als rein geographische Kategorie ohne politische Konnotation vom Altertum dem Mittelalter überliefert worden ist. Europäische Geschichte an das Kriterium zu binden, daß Europa und die Europäer explizit zur Sprache gebracht sind, wäre an sich plausibel, doch haben intensive Forschungen schon seit den 50er Jahren des 20. Jahrhunderts ergeben, daß einschlägige Quellenbelege viel zu spärlich und zu vieldeutig sind, als daß sich (allein) daraus eine Geschichte des europäischen Mittelalters ableiten ließe. Von den Europenses, die gemäß einer vereinzelten spanischen Quelle 732 bei Tours und Poitiers die vordringenden Araber aus dem Feld geschlagen haben, bis zu Enea Silvio Piccolomini, der 1458, fünf Jahre nach der Einnahme Konstantinopels durch die Osmanen, eine Schrift mit dem Titel „Europa“ verfaßt hat, erstreckt sich eine eher disparate Kette von Reminiszenzen an das traditionelle Wissen von den drei Erdteilen Asien, Europa und Afrika, das vor allem in Situationen der äuße- 
ren Bedrohung zu mobilisieren war. Europa war es dann, das sich nach der Meinung belesener Zeitzeugen der Sarazenen, der Wikinger, der Ungarn, der Tataren, schließlich der Türken zu erwehren hatte, doch blieb das im ganzen ein elitärer Sprachgebrauch, ein (wie man gesagt hat) jederzeit abrufbarer "transnationaler Abgrenzungsbegriff“, dem ohne bestimmten Inhalt keine verbreitete und dauerhafte Selbstwahrnehmung, schon gar nicht eine handlungsfähige politische Ordnung entsprach. Es leuchtet ein, daß erst im Zuge der großen Entdeckungen an der Schwelle der Neuzeit Europa in einer allgemein erfahrbaren Weise zu einem Kontinent neben anderen geworden ist.

Andere Begriffe waren im Mittelalter weit besser geeignet, gleichsam von innen heraus zum Ausdruck zu bringen, worin man die gemeinsame Identität gegenüber den eben aufgezählten Fremden und überhaupt gegenüber dem Rest der bekannten Welt erblickte: Christianitas, ecclesia, Occidens, in geringerem Maße auch Latinitas. Damit sind positiv Elemente der Homogenität bezeichnet, die zumindest auf den ersten Blick viel tragfähiger als der blasse Europa-Gedanke für eine die nationalen Divergenzen überwindende Auffassung von der mittelalterlichen Vergangenheit unseres Kontinents erscheinen. Während Latinitas und bis zu einem gewissen Grade auch Occidens (Abendland) die Polarität von römisch-katholischem und griechisch-orthodoxem Kirchentum akzentuierten, kehrten Christianitas und ecclesia eine religiöse Gemeinsamkeit und den Antagonismus zum nichtchristlichen Barbaren- oder Heidentum hervor. Ausgehend von dieser gut bezeugten Selbstdeutung, kam es zu dem modernen Leitbild vom christlichen Mittelalter bzw., bei Beschränkung des Blicks auf den Okzident, vom christlichen Abendland (neuerdings auch gern als Lateineuropa bezeichnet), welches abgesehen von allerlei publizistischen und literarischen Adaptationen auch für wissenschaftlich fundierte Darlegungen einen einladenden Rahmen abgegeben hat und weiterhin abgibt. Zu behandeln sind dann die räumliche Ausbreitung des Christentums vom einstigen römischen Reichsboden aus auf alle Länder Europas, zuletzt 1386 nach Litauen, die Strukturierung des weiten Raumes durch die hierarchische Organisation der Kirche, gipfelnd im Papst in Rom, die Verflechtung der christlichen Mission mit der Entstehung der Nationen, die Ausprägung eines von den Bischöfen gestützten spezifischen Typus von christlicher Monarchie und die Austarierung des Verhältnisses von weltlicher und geistlicher Gewalt, die konkreten Formen der transnationalen Begegnung auf den Kreuzzügen, auf den Konzilien, auf den Pilgerwegen und an den Universitäten. Am Ende steht kurz vor 1500 ein geschichtlicher Augenblick, in dem nach dem Fall von Konstantinopel und nach dem Sieg der spanischen Reconquista, 
aber noch vor den überseeischen Entdeckungen das Christentum ziemlich exakt auf Europa konzentriert erscheint.

Die eingängige und in sich durchaus stimmige Vorstellung von einer im Verlauf des Mittelalters fortschreitenden Gleichsetzung des Kontinents Europa mit der christlichen Welt hat üblicherweise zum Ausgangspunkt das Zeitalter der Karolinger, als die missionarische Expansion des Christentums über die alten römischen Reichsgrenzen an Rhein und Donau hinweg in Gang kam und Karl der Große in seinem fränkischen Imperium eine beachtliche Integrationsleistung vollbrachte, die das Zeitalter der Völkerwanderung überwand und häufig als „Grundlegung der europäischen Einheit im Mittelalter" gerühmt worden ist. Kaum zufällig stammen solche Einschätzungen vornehmlich aus den 50er und den 60er Jahren des vorigen Jahrhunderts, als der beginnende europäische Einigungsprozeß nach historischen Leitbildern verlangte und eine Europäische Wirtschaftsgemeinschaft entstand, deren anfänglicher räumlicher Umfang in verblüffender Weise dem Reich Karls des Großen ähnlich sah. Schon mit der späteren Aufnahme Großbritanniens und Irlands, dann Spaniens und Portugals, vor allem aber Griechenlands haben sich diese Konturen merklich gewandelt, und erst recht die Wende von 1989/90 erlaubt es nicht länger, das historische Selbstbild des zusammenrückenden Kontinents allein von der karolingischen Tradition herzuleiten. Wenn es bei der Leitvorstellung vom christlichen Mittelalter bleiben soll, muß anerkannt werden, daß Byzanz und die griechische Kirche mit gleichem Recht dazugehörten und einen kaum geringeren Anteil als die Lateiner an der fortschreitenden Ausbreitung des Christentums in Europa gehabt haben. So wie Skandinavien, die Westslawen, Ungarn und ein Teil der Südslawen von westlicher Seite „europäisiert" worden sind, haben sich die orthodoxen Byzantiner beim anderen Teil der Südslawen sowie bei den Ostslawen, mithin von Bulgarien bis Rußland, die kirchlich-kulturelle Dominanz gesichert. Auch wenn sich in der Neuzeit bald schon die Gewichte mächtig verschoben, weil das koloniale Ausgreifen in alle Welt allein dem (mittlerweile konfessionell gespaltenen) Lateineuropa zugute kam, während die Orthodoxie nach dem Untergang von Konstantinopel auf eine begrenzte Weltgegend beschränkt blieb, ist für das gesamte Mittelalter unbedingt an der Bipolarität des christlichen Europa festzuhalten.

Allerdings sind seit Jahren auch gegen dieses erweiterte Konzept bedenkenswerte Einwände erhoben worden. Sie beziehen sich zum einen darauf, daß dabei das Judentum ausgeblendet wird, das zwar nicht überall und jederzeit, aber doch das ganze Mittelalter hindurch als religiöse und kulturelle Minderheit inmitten der Christen wie auch der Muslime Europas 
existierte, freilich nirgends eine eigenständige politische Rolle zu spielen vermocht hat. Infolge einer insgesamt relativ ungünstigen Quellenlage, die ihrer Natur nach eher Konfliktsituationen als friedliche Koexistenz hervorkehrt, bestehen nicht geringe Unsicherheiten über den quantitativen Umfang und die ökonomische Bedeutung dieser Minorität, weniger über ihren Einfluß auf das Geistesleben, doch ist fraglos das Postulat berechtigt, die Juden als Faktor von eigenem Gewicht im historischen Erscheinungsbild Europas zu berücksichtigen. Gravierender noch erscheint mir die Kritik, die darauf abzielt, daß Europa im Mittelalter weder von vornherein in allen Teilen christlich gewesen noch dies überall auf die Dauer geblieben ist. Der jahrhundertelange, nicht ohne Rückschläge verlaufene Vorgang der Christianisierung der Mitte, des Nordens und des Ostens unseres Kontinents besagt ja umgekehrt, daß diese religiöse Ausrichtung anfangs, etwa zur Zeit Karls des Großen, bloß auf einen Bruchteil Europas zutraf, im übrigen aber mannigfache Formen des sog. Heidentums vorherrschten. Dazu kommt, daß der Islam seit Beginn des 8. Jahrhunderts auf fast der gesamten Iberischen Halbinsel, später in Sizilien und Teilen Unteritaliens, seit dem 14. Jahrhundert in weiten Bereichen der Balkanhalbinsel auf Kosten des westlichen wie des östlichen Christentums Fuß gefaßt und dort auch politische Herrschaft etabliert hat. Aufs ganze gesehen, ist die mittelalterliche Geschichte Europas also tatsächlich nicht allein vom Christentum (und seiner Ausbreitung), sondern - wenn auch in sehr unterschiedlichen Proportionen - von allen drei monotheistischen Weltreligionen geprägt worden, die sich insgesamt von der polytheistischen Kultur der antiken Griechen und Römer deutlich abhoben.

Während das nach wie vor verbreitete, stets auch sinnstiftende Leitbild vom christlichen Mittelalter (auch bei Einschluß einer jüdischen Komponente) im Grunde mit einem räumlich variablen Europa rechnet, das sich gemäß den Fortschritten der Mission und damit zugleich der Ausbreitung von Schriftkultur mit der Zeit bis zu den äußeren Grenzen des Kontinents ausdehnt und gleichzeitig im Süden gewisse Einbußen gegenüber dem Islam erleidet, geht das Alternativkonzept von einem allzeit gleichbleibenden geographischen Rahmen aus, den spätestens das 18. Jahrhundert endgültig auf den Raum bis zum Ural, zum Kaukasus und zum Bosporus fixiert hat, und sucht ohne Rücksicht auf verbindende Merkmale alles in den Blick zu fassen, was sich dort zwischen 500 und 1500 Geltung verschafft hat. Dabei muß in Kauf genommen werden, daß beträchtliche Teile des Kontinents bis ins Hochmittelalter hinein auf der Kulturstufe der Vorgeschichte verharrten, also keine Schriftquellen hervorbrachten, so daß man auf archäologische und philologische Befunde sowie die sporadische Fernbeobachtung 
aus der christlichen Welt angewiesen ist. Zudem kommen mit dem Islam und Byzanz, in gewissem Sinne auch bereits dem Judentum, historische Kräfte ins Spiel, die anders als das lateinische Christentum des Mittelalters nicht auf den geographischen Rahmen Europas beschränkt waren und daher kaum ohne Ausblicke auf Vorderasien und Nordafrika angemessen zu behandeln sind. Von einem wie auch immer gearteten Wir-Gefühl oder auch nur von kontinuierlicher gegenseitiger Wahrnehmung in diesem multikulturell bestimmten Gesamtraum vom Nordkap bis Sizilien und von Irland bis zur Krim kann schwerlich die Rede sein. Solchen Erschwerungen für eine konsistente Darstellung steht der Vorteil gegenüber, präzise auf die heute bei den Europäern dominierende Vorstellung von ihrem Kontinent nicht so sehr als Träger einer Idee wie vielmehr einem bloß räumlichen Gebilde reagieren zu können, auch wenn an dessen mittelalterlicher Vergangenheit allein noch die Vielfalt, ein gewiß zeitloses historisches Phänomen, demonstriert wird.

Ehe uns das ratlos stimmt, sollten wir uns bewußt machen, daß die Geschichte mit ihrem unendlichen Vorrat an Anknüpfungspunkten eben kein objektiv vorgegebener Maßstab unseres Denkens und Handelns, kein Musterbuch für politische Entscheidungen, sondern stets das ist, was wir aus ihr machen. Sie bietet breiten Raum für Assoziationen und Analogien, um einen Vorgang der Gegenwart zu verorten, der seiner Natur nach historisch beispiellos ist. Die Vereinigung der europäischen Nationen nicht durch gewaltsame Unterwerfung, sondern in freier Selbstbestimmung stellt ja tatsächlich nicht die Rückkehr in ein goldenes, vornationales Zeitalter dar, sondern bedeutet etwas qualitativ Neues, nie Dagewesenes, das anscheinend gerade deshalb nicht ohne Leitbilder auskommt.

\section{Literatur}

Borgolte, M.: Vor dem Ende der Nationalgeschichten? Chancen und Hindernisse für eine Geschichte Europas im Mittelalter, in: Historische Zeitschrift 272 (2001) S. 561-596

Borgolte, M. (Hg.): Unaufhebbare Pluralität der Kulturen? Zur Dekonstruktion und Konstruktion des mittelalterlichen Europa (Historische Zeitschrift, Beihefte 32), München 2001

Borgolte, M.: Europa im Bann des Mittelalters. Wie Geschichte und Gegenwart unserer Lebenswelt die Perspektiven der Mediävistik verändern, in: Jahrbuch für europäische Geschichte 6 (2005) S. 117-135

Borgolte, M.: Ein einziger Gott für Europa. Was die Ankunft von Judentum, Christentum und Islam für Europas Geschichte bedeutete, in: Eberhard, W./Lübke, Ch. (Hg.), Die Vielfalt Europas. Identitäten und Räume, Leipzig 2009, S. 581-590 
Ehlers, J.: Imperium und Nationsbildung im europäischen Vergleich, in: Schneidmüller, B./Weinfurter, St. (Hg.), Heilig - Römisch - Deutsch. Das Reich im mittelalterlichen Europa, Dresden 2006, S. 101-118

Fischer, J.: Oriens - Occidens - Europa. Begriff und Gedanke „Europa“ in der späten Antike und im frühen Mittelalter (Veröffentlichungen des Instituts für europäische Geschichte Mainz, Abt. Universalgeschichte 15), Wiesbaden 1957

Fleckenstein, J.: Die Grundlegung der europäischen Einheit im Mittelalter (urspr. 1986), in: ders.: Ordnungen und formende Kräfte des Mittelalters. Ausgewählte Beiträge, Göttingen 1989, S. 127-145

Geary, P. J.: The Myth of Nations. The Medieval Origins of Europe, Princeton/Oxford 2002 (dt.: Europäische Völker im frühen Mittelalter. Zur Legende vom Werden der Nationen, Frankfurt 2002)

Heimpel, H.: Europa und seine mittelalterliche Grundlegung (urspr. 1949), in: ders.: Der Mensch in seiner Gegenwart. Acht historische Essais, Göttingen ${ }^{2}$ 1957, S. 67-86

Hiestand, R.: „Europa “ im Mittelalter - vom geographischen Begriff zur politischen Idee, in: Hecker, H. (Hg.), Europa - Begriff und Idee. Historische Streiflichter, Bonn 1991, S. 33-48

Karageorgos, B.: Der Begriff Europa im Hoch- und Spätmittelalter, in: Deutsches Archiv für Erforschung des Mittelalters 48 (1992) S. 137-164

Oschema, K.: Der Europa-Begriff im Hoch- und Spätmittelalter. Zwischen geographischem Weltbild und kultureller Konnotation, in: Jahrbuch für europäische Geschichte 2 (2001) S. 191-235

Oschema, K.: Europa in der mediävistischen Forschung - eine Skizze, in: Schwinges, R. Ch./Hesse, Ch./Moraw, P. (Hg.), Europa im späten Mittelalter. Politik - Gesellschaft - Kultur (Historische Zeitschrift, Beihefte 40), München 2006, S. 11-32

von Padberg, L. E.: Die Christianisierung Europas im Mittelalter, Stuttgart 1998

Schneider, R.: Europa im Mittelalter. Wahrnehmungshorizont und politisches Verständnis, in: Marti, R. (Hg.), Europa. Traditionen, Werte, Perspektiven, St. Ingbert 2000, S. 69-93

Schneidmüller, B.: Die mittelalterlichen Konstruktionen Europas. Konvergenz und Differenzierung, in: Duchhardt, H./Kunz, A. (Hg.): „Europäische Geschichte“ als historiographisches Problem (Veröffentlichungen des Instituts für europäische Geschichte Mainz, Abt. Universalgeschichte, Beiheft 42), Mainz 1997, S. 5-24

Segl, P.: Europas Grundlegung im Mittelalter, in: Schlumberger, J. A. /Segl, P. (Hg.): Europa - aber was ist es? Aspekte seiner Identität in interdisziplinärer Sicht (Bayreuther Historische Kolloquien 8), Köln/Weimar/Wien 1994, S. 21-43 